\title{
Prevalence of Age-Related Macular Degenerative Disorder Due to Poor Dietary Habits
}

\section{Siddiqa A*, Khair KU, Hashmi D and Naqvi P}

Khawaja Fareed University of Engineering and Information Technology, Pakistan

*Corresponding author: Ayesha Siddiqa, Khawaja Fareed University of Engineering and Information Technology, Rahim Yar Khan, Punjab, Pakistan, Email: ayeshasiddiqa.ft@gmail. com

\section{Case Report}

Volume 5 Issue 1

Received Date: January 03, 2022

Published Date: January 25, 2022

DOI: $10.23880 /$ aabsc-16000173

\section{Abstract}

Globally, food consumption patterns are changed and trends shift to poor dietary habits leading to poor health and food insecurity. Visual health is normally down after a certain age period or with the progression of individual age but now the trends are observed increased visual disorders in early age periods too. The prevalence of age related macular degenerative disorders are increasing mainly due to diets that are deficient in carotenoids like lutein or other essential nutrients that are necessary to improve visual health. Lutein has the potential to directly absorb and deposit in eye macula that even can restore macular pigment density and recover visual strength. Numerous research has proved that diet naturally rich with carotenoids or supplemented has a substantial beneficial impact against age related macular degenerative disorders in all age groups.

Keywords: Age related macular degenerative disorders; Visual health; Carotenoids

Abbreviations: AMD: Age Related Macular Degeneration.

\section{Introduction}

Age related macular degeneration (AMD) is a degenerative disorder of macula, which is a part of eye retina. It is an ordinary cause of vision loss in the older generation predominantly and less frequent in younger adults [1]. It not only affects the developing countries but the developed countries also came under its recipient ones and also the first cause of irretrievable blindness [2]. Roundabout $8.7 \%$ of the population throughout the world has been facing AMD disorder and the number goes on increasing from 196 million to 300 million from the year 2020 to 2040 [2]. The prevalence of AMDs not only affects the standard of life but it is also a contributing factor in the loss of the Health Care network of the world [3]. There are different stages of AMDs according to the severity of the condition that has different types most commonly; dry and wet age related macular degenerations. More frequently people suffer from dry AMD known as atrophic, the condition in which the macula becomes pencil-thin with the progressing of age. It has 3 stages: early, intermediate and late. Once one reaches late AMD stage it will be irreversible, but ways can be made to protect the remaining sight. The onset of dry AMD took longer than many years. And the wet AMD is also known as neovascular that is a rare type of AMD but it results in faster vision loss. National Eye Institute reported that the late AMD stage happens when abnormal angio flourish in the back of the eye but the positive fact is that the treatment of wet AMD is available [4]. Anti-VEGF (Vascular Endothelial Growth Factor) drug is used to treat wet AMD, other prevention steps include dietary supplements and good nutrition ingestion [3].

Evidence confirmed that the AMD is a progressive disease so its manifestation gets worse with time. Early AMD have generally no significant symptoms, the intermediate stage 
results in mild vision loss and troubleshooting in central vision in light occupancy. While the late AMD condition the person starts seeing the wavy line that is straight and blind sort in central vision and bright colors sight starts diminishing [4]. Commonly contributing factors are smoking and family genetics that is more prompt to cause AMD. Therefore, in such conditions individuals have AMD in genetics suggested to visit the doctor for eye examination regularly before it shows any symptoms [4].

\section{AMD in Relation to Diet}

Diet is a vital factor that affects the modality of AMD risk either it took it towards a positive sideways or towards late AMD i.e., severe stage of AMD. The data of detailed studies showed that a diet which is rich in antioxidants (ascorbic acid, vitamin E, lutein, zeaxanthin) and micronutrients is a contributing factor in lowering the risk of AMD while the food containing a high percentage of trans fat also has to boost factor in AMD risks development [5]. Nowadays, people didn't get stick to one diet and it is rarely found that they were using one food in isolation for a long while. Different approaches have been examined to study a wellcharacterized dietary pattern by scoring mechanism. The Mediterranean diet is one of such approaches it contains fruits, vegetables, vitamins and micronutrient-rich food with no red meat which is a potentially protective factor in lowering AMD risks [6]. Although the trend of the Mediterranean diet has been extended to a vast level to AMD to confirm its impact but still no analytical trials confirmed. It is just preferred because anti-oxidant in the diet prevents the damage due to oxidation and the nutrients in food have more health impact that helps in combating the degenerative AMD disease. However, more clinical trials are needed for the conformity of MD towards the prevention of AMD [5].

\section{Carotenoids}

Carotenoids are fat soluble pigments that are found in living sources such as fungi, algae, fruits, vegetables and also in humans. There are more than six hundred carotenoids are reported with having variant structures and split into carotenes xanthophyll's and lycopene [7]. Each class of the above-mentioned carotenoid carries their particular pigment due to the fact of belonging to tetra terpene family basic chemical structure of carotenoids contain a core carbon chain with single and double, cyclic or acyclic altering bonds and these changing bonds are accountable for their coloring and antioxidant property [8]. Carotenoids are responsible for red, green, or orange hues in plants that can be naturally occurring or can be produced by various biotechnological methods in the laboratories due to their high market in food sector [8].
Carotenoids associated with the human diet are lutein, zeaxanthin, $\alpha$-carotene, $\beta$-carotene. During the research, experts found lutein and zeaxanthin are the most dominating macular pigments as observed under High Performance Liquid Chromatography (HPLC) and UV spectra [9]. Lutein and zeaxanthin are antioxidant carotenoids that are crucial in preserving cell membrane to stabilize it, snuffing out free radicals as they absorb the blue light that lowers the risk of age-related macular degeneration. Some other carotenoids such as $\alpha$-carotene, $\beta$-carotene are also beneficial in reducing the threats of diseases relating to age as aging results in biological and cognitive degradation [7].

Hence, their dietary intake or supplementation would be the key to overcoming this increasing worldwide issue as AMD contributes $8.7 \%$ of world's blindness diseases that researches also reported that this rate has been evolved that the average percentage will get doubled in 2050 [2]. Therefore, it is very important to adequately balance the diet of people by their natural food rich with functional nutrients or supplemented food with carotenoids. Analytical data have given the results of carotene content that is commonly available in fruits and vegetables such as apples contain 100$140 \mu \mathrm{g} / 100 \mathrm{~g}$ lutein and spinach 2047-20300 $\mu \mathrm{g} / 100 \mathrm{~g}$ ) [10].

\section{Role of carotenoid in the prevention of AMD's}

In older people, antioxidants' effectiveness gets retarded as a matter of fact that the biological activity, functioning of the brain and organs get lowered with age due to its ability to fight against the external environment that gets very low [11]. A recent database has reported that an average age of 72 years with a healthy life of 63 years globally [12]. Antioxidant carotenoids have been proved to lower the risk of AMD due to their antioxidant activity as they are more efficient than carotene and alpha-tocopherol and they can fight more effectively and rapidly to oxidative stress and protect from auto-oxidation [13].

Age related macular degeneration can result due to aging but have not the only reasons excessive smoking, diet with low carotenoid contents as well as oxidative stress due to environmental oxidative by-products can also be a reason for this disease [14]. Nevertheless, blue lights of high wavelengths from electronic devices can have worst effects on the retinal cells and cause this disease when a person get excessively exposed to it [15]. As the epithelial cells and photoreceptors don't spread due to their post mitosis, hence they don't contain the DNA detection systems as other cells that are more oxygen sensitive [16]. The sensitivity can damage the cell membrane and natural macular carotenoid which absorb blue light and guard against environmental light induced oxidation the damage causes severe problems 
and result in blurred vision [17]. Hence, the role of these carotenoids is crucial in AMD as research suggests that there is an inverse relationship among both if there is a decrease in the diet of carotenoids there will be an increased risk of AMD vice versa [18]. Carotenoids that have been identified as optical therapy agents and studies have shown the significant beneficial effects as zeaxanthin/lutein $(2 \mathrm{mg} / 10 \mathrm{mg} /$ day/ year). It also improves the person's visual performance by a year other health benefits by lowering oxidative stress. Research suggests that lutein and other provitamin nutrients could be a high chance of effective therapy against agerelated illness $[19,20]$.

Carotenoids especially lutein has a role in the prevention of AMD that improves the content of these carotenoids in the patients diagnosed with the disease as compared to normal people its means that these are being damaged due to the illness [21,22]. Moreover, these carotenoids are antiinflammatory and prevent visual disorders like AMDs that caused severe damage. Various epidemiological studies have proved that in age-related eye diseases controlled macular carotenoid supplemented diets have significant control potential, a multicenter approach [23,24]. Another research evidence reported shows $32 \%$ reduced risk of AMD in people who took high content of zeaxanthin and lutein as compared to those who consumed less amount [25].

Mechanism of action of carotenoids in controlling AMDs.

The macular pigment contains carotenoids mainly the Xanthophylls that are situated right in the inner and outer layer of plexiform from where they get attached to the binders of protein. They are usually present in variant proportions. These are also present in the most sensitive region of the eyes as they absorb the blue UV light up to $460 \mathrm{~nm}$ their adequate presence and are crucial for visual health [26]. They filter the blue light across the retina that aid in visualizing distant image from the eyes. Macular pigment density is also directly related to the performance of eyes and visual health [27]. Thus, age related macular degenerative problems are due to the loss of macular pigments and carotenoids that aid in building macula pigments and controlling its losses. These carotenoids provide that optimal density and protect the macula from getting damaged hence helping in controlling AMDs.

\section{Prevalence of AMD}

Particular research is not conducted on the prevalence of AMD in the people following poor dietary patterns but the various available data shows that the ratio of prevalence of AMD is increasing especially in European countries and the number of patients suffering from any kind of AMD all over the world is elevating day by day that is being controlled by the intake of medicines but it can be suppressed by the use of healthy diet on daily basis. About 196 million are getting affected by it all over the world. It can be controlled with the healthy alteration in diet and lifestyle [28,29].

The onset of AMD and its progression in the body is directly linked with the intake of the diet containing low levels of vitamins, carotenoids, and fatty acid i.e. omega-3. The diet that has a portion of carbohydrates like cereals is refined after processing and loses some of its beneficial constituents that are needed by the body that making the body deficient of those nutrients. Processing vegetables beyond a certain limit results in the destruction of the essential vitamins in them, which will have made it much better to consume the leafy vegetables in raw form. Eating food having high level of sugar like sweets over a certain limit is bad for health similarly consuming food rich in fats and oils adds into the category of unhealthy diet [28]. Poor dietary habits, like consumption of a lot of energy bars, drinks, junk food and fried food, lead to high prevalence of Age-related macular disorders. Therefore, the frequency of AMD is directly proportional to the poor or unhealthy diet. It has been proved through studies that intake of carotenoids mainly lutein and zeaxanthin, vitamin $\mathrm{A} \& \mathrm{C}$ and minerals like $\mathrm{Cu}, \mathrm{Zn}$ through the intake of fruits, vegetables and meat of fish $[28,30]$.

\section{Control of AMDs}

Diet includes any food item having macro \& micro nutrients that should be consumed in daily recommended proportions. People of different areas have different dietary patterns consisting of different nutrients and the nutritional level differs a lot. People consuming a diet consisting of cereal grains, fruits, vegetables, poultry, fish etc. are less likely to diagnose with AMD [31].

Food material having a prominent amount of Vitamin A, B6 \& $\mathrm{C}$ found an association with the lower risk of AMD at the intermediate level [32]. Plant-based diet including nuts and spices etc. is proven beneficial against AMD and reduces oxidative stress. A diet containing refined cereals, red meat, fried products and other such products is not favorable in reducing the chances of AMD. From the category of oils, olive oil is found to help decrease the AMD level in body [31].

Numerous experimental studies have that food containing carotenoids play an effective role against AMDs and cataracts. From the category of carotenoids, lutein and zeaxanthin are present in spinach, kale, asparagus, pistachio, egg, and in many other products. Lutein and Zeaxanthin are the important constituents of Macular pigment in the retina [33]. It plays a vital role in maintaining eye health, improvising the functioning of the eye and preventing macula from harmful rays and other free radicals. A diet that has these natural lutein sources in it, increased the efficacy against AMD in its preventive formula. Therefore, to control 
AMD, it is significant to add the food categories such as leafy vegetables, eggs and nuts in your daily dietary pattern. Such food will defend the body against the early and late effects of AMD [3].

\section{Conclusion}

Conclusively, the relation of the prevalence of AMD with the dietary habits of people has found a strong association of AMD risk with poor dietary habits. The communities following healthy dietary patterns are resulted to be at lower risk of AMD but the poor dietary pattern resulted at higher risk of AMD. The dietary habits of consuming high calories and an unbalanced diet accelerates the progression of Agerelated macular diseases in all age groups. Therefore, the balanced and supplemented diet that has a positive impact on body metabolism works efficiently to improve the immune system, antioxidant potential and visual health that will control all age-related and visual disorders.

\section{References}

1. Waugh N, Loveman E, Colquitt J, Royle P, Yeong JL, et al. (2018) Treatments for dry age-related macular degeneration and Stargardt disease: a systematic review. Health Technology Assessment 22(27): 1-196.

2. Wong WL, Su X, Li X, Cheung CMG, Klein R, et al. (2014) Global prevalence of age-related macular degeneration and disease burden projection for 2020 and 2040: a systematic review and meta-analysis. The Lancet Global Health 2(2): 106-116.

3. Rinninella E, Mele MC, Merendino N, Cintoni M, Anselmi G, et al. (2018) The role of diet, micronutrients and the gut microbiota in age-related macular degeneration: New perspectives from the gut-retina axis. Nutrients 10(11): 1677.

4. Sabeti F, Lane J, Rohan EMF, Essex RW, McKone E, et al. (2021) Relationships between retinal structure and function and vision-related quality of life measures in advanced age-related macular degeneration. Graefe's Arch Clin Exp Ophthalmol 259(12): 3687-3696.

5. Woodside JV, Cole D, Hogg RE (2019) Interconnecting the Mediterranean Diet and Age-Related Macular Degeneration, in Handbook of Nutrition, Diet, and the Eye. Elsevier pp: 425-438.

6. Hogg RE, Woodside JV (2019) Mediterranean diet and age-related macular degeneration: is it time to attempt dietary modification? Ophthalmology 126(3): 391-392.

7. Wu J, Cho E, WillettWC, Sastry SM, Schaumberg DA (2015)
Intakes of lutein, zeaxanthin, and other carotenoids and age-related macular degeneration during 2 decades of prospective follow-up. JAMA Ophthalmology 133(12): 1415-1424.

8. Milani A, Basirnejad M, Shahbazi S, Bolhassani A (2017) Carotenoids: biochemistry, pharmacology and treatment. Br J Pharmacol 174(11): 1290-1324.

9. Bone RA, Landrum JT, Tarsis SL (1985) Preliminary identification of the human macular pigment. Vision Res 25(11): 1531-1535.

10. Laura A, Alvarez Parrilla E, González Aguilar GA (2009) Fruit and vegetable phytochemicals: chemistry, nutritional value and stability. John Wiley \& Sons.

11. Navaratnarajah A, Jackson SH (2017) The physiology of ageing. Medicine 45(1): 6-10.

12. Organization WH (2019) Global Health Observatory (GHO) data, Life Expectancy 2019.

13. Miller NJ, Sampson J, Candeias LP, Bramley PM, Rice Evans CA, et al. (1996) Antioxidant activities of carotenes and xanthophylls. FEBS letters 384(3): 240-242.

14. Chen Y, Bedell M, Zhang K (2010) Age-related macular degeneration: genetic and environmental factors of disease. Mol Interv 10(5): 271-281.

15. Tosini G, Ferguson I, Tsubota K (2016) Effects of blue light on the circadian system and eye physiology. Mol Vis 22: 61-72.

16. Blasiak J, Petrovski G, Veréb Z, Facskó A, Kaarniranta $\mathrm{K}$ (2014) Oxidative stress, hypoxia, and autophagy in the neovascular processes of age-related macular degeneration. BioMed Res Int 2014: 768026.

17. Bernstein PS, Li B, Vachali PP, Gorusupudi A, Shyam R, et al. (2016) Lutein, zeaxanthin, and meso-zeaxanthin: The basic and clinical science underlying carotenoid-based nutritional interventions against ocular disease. Prog Retin Eye Res 50: 34-66.

18. van Asten F, Simmons M, Singhal A, Keenan TD, Ratnapriya R, et al. (2018) A deep phenotype association study reveals specific phenotype associations with genetic variants in age-related macular degeneration: Age-Related Eye Disease Study. 125(4): 559-568.

19. Bovier ER, Renzi LM, Hammond BR (2014) A doubleblind, placebo-controlled study on the effects of lutein and zeaxanthin on neural processing speed and efficiency. PloS one 9(9): e108178. 
20. Yao Y, Qiu QH, Wu XW, Cai ZY, Xu S, et al. (2013) Lutein supplementation improves visual performance in Chinese drivers: 1-year randomized, double-blind, placebo-controlled study. Nutrition 29(7-8): 958-964.

21. Bone RA, Landrum JT, Mayne ST, Gomez CM, Tibor SE, et al. (2001) Macular pigment in donor eyes with and without AMD: a case-control study. Invest Ophthalmol Vis Sci 42(1): 235-240.

22. Bone RA, Landrum JT, Dixon Z, Chen Y, Llerena CM (2000) Lutein and zeaxanthin in the eyes, serum and diet of human subjects. Exp Eye Res 71(3): 239-245.

23. Cho E, Hankinson SE, Rosner B, Willett WC, Colditz GA (2008) Prospective study of lutein/zeaxanthin intake and risk of age-related macular degeneration. Am J Clin Nutr 87(6): 1837-1843.

24. Moeller SM, Parekh N, Tinker L, Ritenbaugh C, Blodi B, et al. (2006) Associations between intermediate agerelated macular degeneration and lutein and zeaxanthin in the Carotenoids in Age-related Eye Disease Study (CAREDS): ancillary study of the Women's Health Initiative. Arch Ophthalmol 124(8): 1151-1162.

25. Ma L, Dou HL, Wu YQ, Huang YM, Huang YB, et al. (2012) Lutein and zeaxanthin intake and the risk of age-related macular degeneration: a systematic review and metaanalysis. Br J Nutr 107(3): 350-359.

26. Sabour Pickett S, Nolan JM, Loughman J, Beatty S (2012) A review of the evidence germane to the putative protective role of the macular carotenoids for agerelated macular degeneration. Mol Nutr Food Res 56(2): 270-286.

27. Fletcher LM, Engles M, Hammond BR (2014) Visibility through atmospheric haze and its relation to macular pigment. Optom Vis Sci 91(9): 1089-1096.

28. Francisco SG, Smith KM, Aragonès G, Whitcomb EA, Weinberg J, et al. (2020) Dietary Patterns, Carbohydrates, and Age-Related Eye Diseases. Nutrients 12(9): 2862.

29. Jong EK, Geerlings M, Hollander A (2020) Age-related macular degeneration. Genetics and Genomics of Eye Disease pp: 155-180.

30. Li JQ, Welchowski T, Schmid M, Mauschitz MM, Holz FG (2020) Prevalence and incidence of age-related macular. Br J Opthalmol 104(8): 449-450.

31. Chapman NA, Jacobs RJ, Braakhuis AJ (2019) Role of diet and food intake in age-related macular degeneration: a systematic review. Clin Exp Ophthalmol 4(1): 106-127.

32. Agron E, Mares J, Clemons TE, Swaroop A, Chew EY (2021) Diertary nutrient intake and progression to late age-related macular denegeneration in the age-related eye disease studies 1 and 2 Ophthalmol 128(3): 425442 .

33. vel Sobczuk IK, Święch A (2021) The role of balanced supplementation in diet of age-related macular degenration patients. 8(1): 19-25. 